\title{
From the space-time model to the time-invariant space model
}

\author{
Amrit S. Šorli \\ Bijective Physics Institute \\ sorli.bijective.physics@gmail.com
}

\begin{abstract}
Carlo Rovelli's research on time suggests that time has no physical existence, that it is an illusion. Bijective research confirms Rovelli is right. Time is what we measure with clocks. We measure with clocks the numerical sequential order of material change, i.e. the motion running in time-invariant space. Time as the duration of change enters existence only when measured by the observer. The change runs only in time-invariant universal space. Humans are experiencing a run of changes in time-invariant space in the frame of the linear psychological time "past-present-future" that has its basis in the neurological activity of the brain. In the universe, there is neither a physical past nor physical future. There exists only what we can observe with our senses and measure with apparatuses.
\end{abstract}

Keywords: Space; Time; Change; Block universe; Psychological time; Bijectivity principle.

\section{Introduction}

Carlo Rovelli suggested that time is an illusion. On the other hand, he is not categorically denying the mainstream view that time cannot be eliminated from physics: "On the other hand, I also see well that the view I present here is far from being uncontroversial. Several authors maintain the idea that the notion of time is irreducible, and cannot be eliminated from fundamental physics" [1]. We will show in this article Rovelli is right about time being an illusion but still, time that we measure with clocks can remain in physics.

We introduce a model of time, which is an exact model of the time running in physical reality, using a bijective research methodology which is based on experimental results. There are no theoretical assumptions on time based on speculation, as for example what has been assumed for more than 100 years about time as the $4^{\text {th }}$ dimension of space. There is no single experimental data confirming this last view.

Let's take the following example: a photon is moving in space from point $\mathrm{A}$ to point $\mathrm{B}$. The distance $d$ between A and B can be expressed as the sum of Planck lengths:

$$
d=d_{P 1}+d_{P 2}+\cdots+d_{P n}=\sum_{i=1}^{n} d_{P i}
$$


Photon is moving from $d_{P 1}$ to $d_{P 2}$ and so on; every Planck distance $d_{P n}$ corresponds exactly to one Planck time $t_{P}$. In this sense, the Planck time is the fundamental unit of photon sequential motion from one to the next Planck distance. We can therefore write:

$$
\begin{gathered}
t=t_{P 1}+t_{P 2}+\cdots+t_{P n}=\sum_{i=1}^{n} t_{P i} \\
\frac{\sum_{i=1}^{n} d_{P i}}{\sum_{i=1}^{n} t_{P i}}=c
\end{gathered}
$$

Photon is moving only in space and not in some physical time; the duration of photon motion from A to B in space is the sum of Planck times. Time is not continuous, is a discrete quantity; it is not some physical quantity that is running on its own, but the epiphenomenon of change, i.e. motion, its sequential numerical order. Time as the numerical sequential order of changes is a fundamental time; when fundamental time is measured by the observer, duration enters in existence. Duration is the so-called emergent time; there is no emergent time without the measurement of the fundamental time [2].

We introduce a new research methodology, named bijectivity principle: let's consider the universe as a set $X$. In this set, we have four fundamental elements:

a) the energy in different forms: universal space is an energy structure, electromagnetic energy is a type of energy, dark energy is a type of energy, dark matter is a type of energy, energy in the form of matter;

b) the change;

c) the time;

d) the observer.

To build an adequate model of the universe in the set $Y$, which is the model of the universe, we must have there the same fundamental elements (Figure 1).

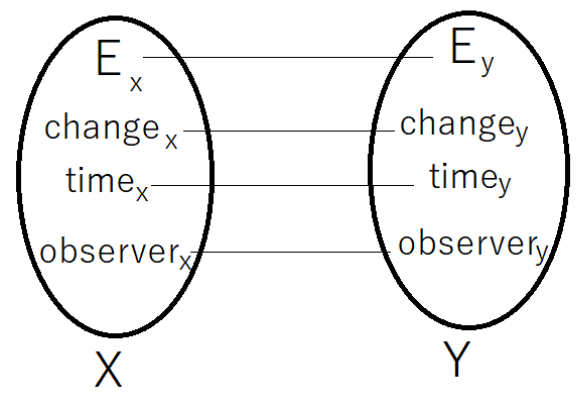


Figure 1: Bijective model of the universe.

$$
\begin{aligned}
& X:\left\{E_{x}, C_{x}, t_{x}, O_{x}\right\} \quad(4 \mathrm{a}), \\
& Y:\left\{E_{y}, C_{y}, t_{y}, O_{y}\right\} \quad \text { (4b), }
\end{aligned}
$$

where $E_{x}, C_{x}, t_{x}, O_{x}$ are energy, change, time and observer in the physical universe, and $E_{y}$, $C_{y}, t_{y}, O_{y}$ the corresponding quantities in the model of the universe. We can write the following equation:

$$
f\left(t_{x}\right)=t_{y}
$$

The time in the model of the universe $t_{y}$ is related to the time $t_{x}$ in the physical universe by the bijective function of set theory. Bijectivity principle assures an adequate model of the universe; the physical universe and the corresponding model of the universe are related by a bijective function. In this bijective model of the universe, time in the physical universe has exactly the same meaning in the model of the universe: time is the sequential numerical order of material change, i.e. motion. Time is not continuous, it is discrete; Planck time is the fundamental unit of time.

The idea that space and time should be defined on the basis of set theory is not new. It was already proposed back in 1997 by Costa, Bueno, and French: "Science is the search for structure. Such a bold claim certainly deserves further elaboration and justification, but it is one which, it has recently been emphasised, has been held by a number of scientists and philosophers. Our aim in this note is two-fold. First, we wish to relate the consideration of such an aim to a long standing programme of work which has sought to develop a mathematically precise treatment of the notion of structure itself. In doing so, we shall analyse a particular illustrative case and thereby generate favourable evidence for the above claim as a whole. Thus, we shall consider the problem of constructing a set-theoretic structure, in Suppes's sense, which is capable of providing an axiomatic basis for those notions of space and time which underpin various theories of physics" [3].

We use in our research the bijectivity principle of set theory; the result is that the universal space is a time-invariant energy structure where time is the duration of material changes, i.e. motion. This model fits experimental physics and is in agreement with the mathematical formalism of Special Relativity and with the neuroscience research results on time. 


\section{Neuroscience confirms linear time "past-present-future" exists in the brain}

Time as some physical quantity that is running in the universe was never observed experimentally and represents a "hard problem" of physics. In order to understand time, and to build an adequate model of it, we have to understand how we experience change, i.e. motion. The human being perceives with senses the information about the change. The information is transformed into an electromagnetic signal and moves through nerves to the sight center in the brain. Here there is a neuronal activity that is continuously creating the psychological sensation of linear time: past, present, future. Linear time "past-present-future" is the result of neuronal activity of the brain. Several researches confirm that animal and human experience of linear time has the origin in neuronal activity of the brain $[4,5,6]$. We experience the material change, i.e. the motion in the frame of psychological time, which has its basis in neuronal activity; that is why we see changes running in some physical time despite there is no physical time in the universe (Figure 2).

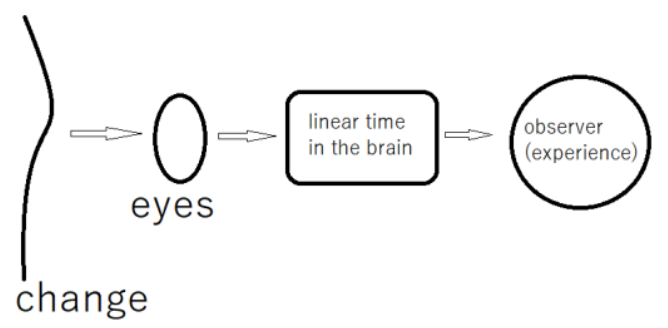

Figure 2: Linear time "past-present-future" is only in the brain.

Einstein was aware of the psychological time; he used to say: Past, present and future are stubbornly persistent illusions. Time has no independent existence apart from the order of events by which we measure it." [7]. In Minkowski manifold, the arena of Einstein's Special Relativity, the $4^{\text {th }}$ dimension is not time, but is a spatial coordinate $X_{4}=i c t$, i.e. the product of the imaginary number $i$, the speed of light $c$ and the time $t$ as the numerical sequential order of the change. Minkowski manifold has not dimension $3 D+t$; it is $4 D$. In this four-dimensional continuum, time is the sequential numerical order of events.

Recent research confirms that by zapping the areas of visual cortex V5 with magnetic field, the duration of a given event that people observe cannot be well estimated. The duration of a given motion is defined by the neuronal activity of the visual cortex. When functioning of the visual cortex area is disturbed by the magnetic field, the experience of motion is disturbed too [8]. 


\section{4. "Block universe" model development with time-invariant space}

The time-invariant space is the underlying reality of the universe. It has different names: "the non temporal and non spatial underlying reality [9], to the "timeless configuration space"[10], or "superfluid quantum vacuum" [11]. In this non temporal underlying reality, time travels are categorically excluded [12].

One can travel only in this underlying reality and time is the duration of its motion. In this perspective Minkowski manifold is only a theoretical model that describes this underlying time-invariant reality. The formalism $X_{4}=i c t$ confirms that time $t$ is the duration of photon motion. $4^{\text {th }}$ dimension $X_{4}$ is not time $\left(X_{4} \neq t\right)$ and it is not "temporal", it is spatial in the same way as $X_{1}, X_{2}, X_{3}$. It was a historical mistake that Mikowski manifold was named "space-time". Minkowski manifold is four-dimensional geometry where time $t$ is an element of the $4^{\text {th }}$ dimension $X_{4}$. The product of time $t$ as the duration and light speed $c$ is spatial distance $d: d=$ $c t$. Einstein has added to this spatial distance imaginary number $i$, so we can write $X_{4}=i d$. This imaginary distance in the Minkowski model has no bijective correspondence with the physical world. Equation $f: i d_{X} \rightarrow i d_{Y}$ is false. Imaginary distance $i d$ cannot exist in the physical world. In Special Relativity the space-time interval $S$ has been defined as $S^{2}=$ $c^{2} t^{2}-\left(X^{2}+Y^{2}+Z^{2}\right)$. With the introduction of natural units: $c=\hbar=1$, the space-time interval becomes: $S^{2}=t^{2}-\left(X^{2}+Y^{2}+Z^{2}\right)$ and the fourth coordinate of Minkowski manifold was interpreted as time $t$. The fourth coordinate of Minkowski manifold in its original form $X_{4}=i c t$ turned into $X_{4}=t$. Time has been fully merged with space and still today we think that time is $4^{\text {th }}$ dimension of space-time. For a century it was thought this is one of the most important achievements of physics. Today this achievement is questionable. We have to admit that rigorous bijective mathematical analysis confirms that time $t$ in Minkowski manifold represents the duration of photon motion in time-invariant space; not more and not less.

The idea that time is the $4^{\text {th }}$ physical dimension of the universal space is one of the main obstacles to physics progress. Universal space is time-invariant, there is no physical past and there is no physical future. Material change run in time-invariant space. This is the bijective development of the "block universe" model where past and future are coexisting: "A new challenge emerged a century ago with Minkowski's space-time as it provided support for viewing the universe as a four-dimensional space-time block that exists as one entity. In this view, called the block universe (or eternalism in philosophical discussions), there is no basis for singling out a present time that separates the past from the future because all times coexist 
with equal status" [13]. In the bijective model of the "block universe" past and future are not coexisting in some eternal atemporal reality, they are non-existent. Irreversible universal change run in time-invariant space. When change $X+1$ enters existence, the change $X$ is not existing anymore. When change $X+2$ is entering existence, the change $X+1$ is not existing anymore. What really exists in the universe is what we can observe with our senses and measure with apparatuses. All the rest is pure imagination that is out of the frame of the rigorous bijective model of the universe. Symmetry in time that is represented by the formula $T: t \rightarrow$ $-t$ is the model that has no bijective correspondence with the physical world. In the universe, there is no symmetry in time because time is not a physical dimension in which the universe exists. Time is the duration of changes in time-invariant space of the "block universe".

\section{Discussion}

In physics, we experience the increase of entropy and run of physical changes through the linear psychological time of "past-present-future"; we experience that changes are running in some linear time. Entropy increasing in time is the wrong imagination based on our experience of material changes run in the frame of psychological time. Considering that universal space in the perspective of bijective physics is timeless, the universe runs only in this time-invariant space and not in time. With clocks, we measure duration of events in time-invariant space. The arrow of time exists only in the form of mathematical arrow of the sequential numerical order of universal changes running in a time-invariant space. The entropy of a given system is increasing only in space and not in time; time is the numerical sequential order of entropy increasing.

The idea that time has no physical existence and that with clocks we measure internal relations between different physical changes running in a timeless space is entering in the mainstream of physics [14], so as the idea that gravity does not require time and is encoded in a timeless configuration [15]. Time-invariant universal space is the direct information medium of entanglement by EPR-type phenomena [16]. For a century, entanglement has been difficult to understand because of a wrong image, i.e. that space-time (where time is a $4^{\text {th }}$ physical dimension of space) is the fundamental arena of the universe.

We show in this article, universal changes are running in space that is time-invariant. Humans, we experience this time-invariance of universal space as "Now". Albert Einstein used to say: "that there is something essential about the Now which is just outside the realm of science" 
[17]. Neuroscience results that linear time "past-present-future" is created by the neuronal activity of the brain is bringing Einstein's Now into physics. Rovelli is right, time is an illusion in the sense, it has no physical existence. The mainstream did not understand well yet Rovelli's discovery [18]. What is important to understand is that considering physical time is an illusion, clocks are remaining the useful tools for measuring the numerical sequential order of changes in time-invariant space.

\section{Conclusions}

A bijective research methodology confirms that time is not an illusion. Time is what we measure with clocks. With clocks, we measure duration material changes, i.e. motion that runin time-invariant space of the "block universe". We experience the flow of material changes, i.e. motion in the frame of linear psychological time "past-present-future" that has the origin in the neuronal activity of the visual cortex.

\section{References:}

1. Rovelli, C. "Forget time". Found Phys 41, 1475 (2011). https://doi.org/10.1007/s10701-011-9561-4.

2. Fiscaletti, D., Sorli, A.S. Perspectives of the numerical order of material changes in timeless approaches in physics. Found. Phys. 45(2), 105-33 (2015). https://doi.org/10.1007/s10701-014-9840-y.

3. da Costa, N.C.A., Bueno, O. \& French, S. Suppes Predicates for SpaceTime. Synthese 112, 271-279 (1997). https://doi.org/10.1023/A:1004984927979.

4. Buhusi, C., Meck, W. What makes us tick? Functional and neural mechanisms of interval timing. Nat. Rev. Neurosci. 6, 755-765 (2005). https://doi.org/10.1038/nrn1764.

5. Ivry, R.B., Spencer, R.M. The neural representation of time. Curr. Opin. Neurobiol. 14(2), 225-232 (2004). doi:10.1016/j.conb.2004.03.013.

6. Mauk, M.D., Buonomano, D.V. The neural basis of temporal processing. Annu. Rev. Neurosci. 27, 307-340 (2004). doi:10.1146/annurev.neuro.27.070203.144247.

7. Sorli, A.S., Klinar, D., Fiscaletti, D. New insights into the special theory of relativity. Phys. Essays 24(2), 313-318 (2011). doi: 10.4006/1.3590161.

8. Protopapa, F., Hayashi, M.J., Kulashekhar, S., van der Zwaag, W., Battistella, G., Murray, M.M., Kanai, R., Bueti, D. Chronotopic maps in human supplementary motor area. PLoS Biol. 17(3), e3000026 (2019). https://doi.org/10.1371/journal.pbio.3000026.

9. Diederik, A., Relativity Theory Refounded (2015). https://arxiv.org/abs/1511.08735.

10. Małkiewicz, P. Internal clocks in timeless universe. J. Phys. Conf. Ser. 880, 012046 (2017). https://iopscience.iop.org/article/10.1088/1742-6596/880/1/012046.

11. Šrli, A.S. Mass-Energy Equivalence Extension onto a Superfluid Quantum Vacuum. Sci. Rep. 9, 11737 (2019). https://doi.org/10.1038/s41598-019-48018-2. 
12. Fiscaletti, D., Sorli, A. Bijective Epistemology and Space-Time. Found Sci 20, 387-398 (2015). https://doi.org/10.1007/s10699-014-9381-z.

13. Vaccaro, J.A. The quantum theory of time, the block universe, and human experience. Phil. Trans. R. Soc. A. 376, 20170316 (2018). http://doi.org/10.1098/rsta.2017.0316.

14. Barbour, J. The Nature of Time. arXiv:0903.3489v1 [gr-qc] (2009). https://arxiv.org/abs/0903.3489.

15. Gomes, H. Quantum gravity in timeless configuration space. Classical Quant. Grav. 34(23) (2017). https://iopscience.iop.org/article/10.1088/1361-6382/aa8cf9.

16. Fiscaletti, D., Sorli, A.S. Searching for an adequate relation between time and entanglement. Quantum Stud.: Math. Found. 4(4), 357-374 (2017). https://link.springer.com/article/10.1007/s40509-017-0110-5.

17. Smolin, L. Time Reborn: From the Crisis in Physics to the Future of the Universe, Houghton Mifflin Harcourt (2013), p. 93 ss.

18. Jaffe, A. The illusion of time. NATURE 556, 304-305 (2018). https://www.nature.com/articles/d41586-018-04558-7. 